\title{
Music in a bag? Controlling the bag of Majorcan and Galician bagpipes
}

Augustin Ernoult, Benoît Fabre, Cassandre Balosso-Bardin, Ilya Franciosi, and Patricio de la Cuadra

Citation: The Journal of the Acoustical Society of America 142, 1454 (2017); doi: 10.1121/1.5002692

View online: http://dx.doi.org/10.1121/1.5002692

View Table of Contents: http://asa.scitation.org/toc/jas/142/3

Published by the Acoustical Society of America 


\title{
Music in a bag? Controlling the bag of Majorcan and Galician bagpipes
}

\author{
Augustin Ernoult ${ }^{\mathrm{a})}$ and Benoît Fabre \\ Lutherie-Acoustique-Musique, Institut Jean le Rond d'Alembert, Unité Mixte de Recherche UMR 7190, \\ CNRS, Université Pierre et Marie Curie Univ Paris 06, Sorbonne Universités, Paris, F-7500, France \\ Cassandre Balosso-Bardin \\ Chaire GeAcMus, Sorbonne Universités, University of Lincoln, Lincoln, United Kingdom \\ Ilya Franciosi \\ Chaire GeAcMus, Sorbonne Universités, Paris, France \\ Patricio de la Cuadra \\ Chaire GeAcMus, Sorbonne Universités, Pontificia Universidad Católica de Chile, Santiago, Chile
}

(Received 24 May 2017; revised 21 July 2017; accepted 28 August 2017; published online 19 September 2017)

\begin{abstract}
After defining the mechanical framework of the bag control of bagpipe, this paper presents a study of the bag pressure control in a musical context through the comparison of six players and two bagpipes: one Galician (gaita) and one Majorcan (xeremies), the latter mainly differentiated organologically by a much larger bag size. General observations first lead to the identification and interpretation of the range of control parameters observed. A more detailed analysis of the control parameters during the production of steady notes highlights the coordination between insufflations and the arm displacement necessary to produce a stable and continuous sound. Finally, the bag pressure variation is observed in a musical context and correlated with the musical task, thus, associating different control strategies to the different bagpipes played by the musicians.
\end{abstract}

(C) 2017 Acoustical Society of America. [http://dx.doi.org/10.1121/1.5002692]

[AM]

Pages: $1454-1465$

\section{INTRODUCTION}

Despite their many organological and esthetical differences, bagpipes around the world all function on the same basic principle. Air is insufflated into the bag through a blowpipe or a set of bellows. The arm on the bag then exerts enough pressure to activate the reeds, which drive the vibration of the air column in the different pipes. The air supply, the bag, and the reeded pipe(s) are the common denominators of the instrument. The lungs or the bellows provide the initial source of air injected into the bag. Thanks to its mechanism, bagpipes are able to create a continuous sound. Repertoires, scales, and registers vary according to the instruments and their musical cultures, going from a diatonic scale within a range of a sixth (such as the Greek tsampouna or the Tunisian mizwid) to a fully chromatic scale over two octaves (the uilleann pipes from Ireland). ${ }^{1,2}$

This study focuses on the interaction between the musician and the instrument. Research on this topic has been carried out on other wind instruments, such as the flute, ${ }^{3,4}$ or the brass instruments. ${ }^{5}$ Bagpipes, on the other hand, have the advantage of having an external set of "lungs" which simplifies the acquisition of experimental data. The study of such an instrument is therefore an opportunity to study the player's control of the air supply during a musical performance. The observations made on the control of the bag could be extended to the supply control of other musical

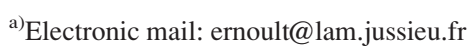

instruments with external supply system such as the accordion, but also in a broader sense to the control of wind instruments and the singing voice.

The main goal of this paper is to characterize the control of the bag. This will inform on how the musician reacts to the instrument. It will reveal which aspects of control are imposed by the behavior of the instrument and which ones respond to the musical context.

To identify pertinent aspects of the control, this study proposes to compare two different cultures and, therefore, two different instruments, played by musicians with different levels of expertise, from beginners to expert players.

\section{A. Instruments studied}

Galician and Majorcan bagpipes (gaita and xeremies, respectively) share many organological characteristics. Both are mouth blown western bagpipes with one conical melodic pipe fitted with a double reed and three cylindrical drones with single reeds. The instruments are supplied in air by the musician insufflating through a blowpipe fitted with a valve in order to prevent the air from escaping once in the bag. Both modern versions of the instruments are similar enough for Majorcan pipers to occasionally use reeds made by Galician makers. However, both present enough differences to justify a comparative study between the two instruments.

Aside from some small differences on the radii and conicity of the melodic pipe, Galician and Majorcan bagpipes differ mainly by the size and the shape of their bag (Fig. 1). Majorcan bagpipes have a much larger bag with the 
drones hanging at the front of the bag, creating a considerable amount of counterweight for the musician. Musically, Galician musicians use a more extensive range, play repertoire that goes from major to minor, and play with effects such as musical pauses during which the musician stops the sound of the melodic pipe and the drones thanks to a specific gesture (Sec. VC). Majorcan musicians play on an instrument that has a range of an octave. Their repertoire is mainly local and retains its characteristics with major/minor changes occurring only in more recent compositions. Majorcan music has mainly been performed in functional and/or local contexts with the traditional pipe and tabor (flabiol and tamboril). Although Galician bagpipes are performed in these contexts, some musicians have developed extremely virtuoso soloist abilities.

As the main organological differences are linked to the bag, the comparison of the control of these instruments appears to be an ideal starting point to study the bag control and its influence on the musical performance.

In Sec. II a simple model of the bag is presented. The experimental setup is discussed in Sec. III, followed by the analysis of some characteristics of the instruments in Sec. IV where the playing by musicians with different levels of expertise is compared. A study of the fine control that musicians from both cultures exert on the bag pressure while interpreting musical excerpts is presented in Sec. V to conclude with an overall analysis and perspectives.

\section{MODEL OF THE INSTRUMENT}

In a first approximation, the mechanical behavior of the bag of the bagpipes can be described by a general model. The aim of this model is to define a mechanical framework allowing the identification of the different control parameters and their link. Simplifying hypotheses are therefore considered in order to highlight these links.

\section{A. Relationship between mechanical parameters}

First assumption is that the bagpipe is made with a membrane with no elasticity. The influence of the bag's material is therefore not taken into account. The forces considered on the bag are the effort exerted by the player's arm and the air pressure. Under this assumption the bag can be modeled by a simple piston as represented in Fig. 2. The surface of the

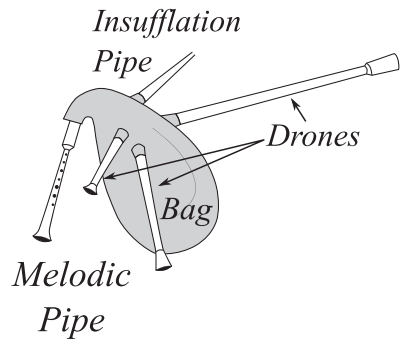

(a)

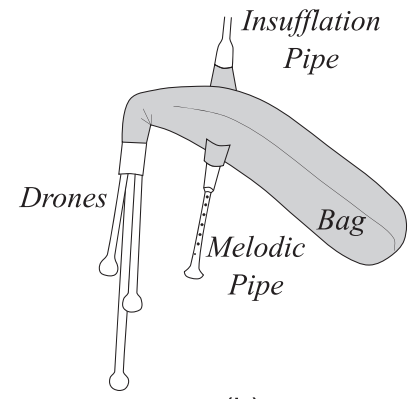

(b)
FIG. 1. Sketch of the studied bagpipes. The relative scale is more or less the same for both instruments. (a) Galician bagpipe "gaita" (bag $\approx 9 \mathrm{~L}$ ). (b) Majorcan bagpipe "xeremies" (bag $\approx 15 \mathrm{~L})$.

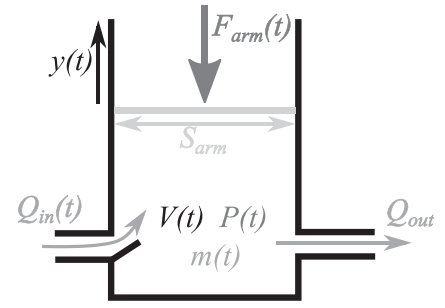

FIG. 2. Sketch of the model of bagpipe.

piston at each instant equals the contact surface $S_{\text {arm }}$ between the bag and the arm of the musician.

The bag is characterized by its inner volume $V$, its inner pressure $P$, and the mass of the inner gas $m$. These three parameters are theoretically linked, knowing the temperature $T$, through the equation of state of the gas. The principal aim of the musician is to control the bag pressure, which influences the sound characteristics such as pitch accuracy, loudness, timbre. ${ }^{6,7}$ The inner pressure can be directly related to the strength of the arm on the bag $F_{\text {arm }}$ with the contact surface $S_{\text {arm }}$ between the moving arm of the musician and the bag of the instrument

$$
P=F_{\text {arm }} S_{\text {arm }}
$$

The control of the pressure variations can also be interpreted as the combined control of the mass and the volume variations. If the variation of the pressure is fast enough, the induced elevation of the inner temperature cannot be compensated by the exchange of thermal energy with outside. Under this condition and adding the assumption of a perfect gas, the transformation of the gas is described as adiabatic and reversible, which is written as follows:

$$
\frac{d P}{P}=\gamma\left(\frac{d m}{m}-\frac{d V}{V}\right)
$$

with $\gamma$ the adiabatic index. The variation of the mass $d m$ is determined by the inflow $Q_{\text {in }}$ brought by the musician into the bag during the performance through the insufflation pipe, and the outflow $Q_{\text {out }}$ exiting the bag through the different pipes (melodic pipe and drones)

$$
d m=\left(Q_{\text {in }}-Q_{\text {out }}\right) \rho(P),
$$

with $\rho(P)$ the volumic mass of the air, function of the bag pressure. The outflow depends a priori on the number of sounding pipes, the type of reeds, and their mechanical characteristics, and also on the inner pressure and the pitch of the notes played..$^{8-10}$

According to the simplified model, the volume variations are controlled by the position $y$ of the musician's arm (Fig. 2)

$$
d V=d y S_{\mathrm{arm}} .
$$

To simplify the interpretations, for a given bagpipe and musician, the temporal variation of the contact surface $S_{\text {arm }}$ and the temporal variation of the outflow $Q_{\text {out }}$ are supposed negligible compared to the variation of the inflow and the arm displacement. They are therefore assumed to be independent of time. Furthermore, as the bag pressure fluctuates within a 
limited range (Sec. IV B), the air density is assumed to be independent of the bag pressure. Under these assumptions, the control of the bag pressure can be summarized as

$$
\frac{d P(t)}{P(t)} \propto \frac{1}{V(t)}\left[\left(Q_{\text {in }}(t)-Q_{\text {out }}\right)-S_{\text {arm }} d y(t)\right] .
$$

With this formula, the bag's pressure control appears to be ensured by a good arm-inflow coordination. This hypothesis gives the musician two control parameters to induce bag pressure variations: the displacement of the arm $d y$ and the inflow $Q_{\text {in. }}$

\section{B. Maintaining a constant bag pressure}

If the musician wants to maintain a constant bag pressure during insufflations $(d P=0)$, he needs to exactly compensate the mass variation by the volume variation

$$
d y(t)=\frac{d m}{\rho S_{\mathrm{arm}}} .
$$

In particular, if the inflow during insufflations is big enough to fill the bag $\left[Q_{\text {in }}(t)>Q_{\text {out }}\right]$, the sign of the mass variation changes during the insufflations: $d m>0$ during insufflations and $d m<0$ otherwise. To keep the bag pressure constant, the variation of mass and the arm displacement must be perfectly coordinated and change signs at the same time, meaning that the player needs to keep a constant force of the arm on the bag while letting the bag inflate during insufflation. If this coordination is not good enough, it will induce a variation of the bag pressure.

\section{Influence of the bag size}

The simplified model of the bag presented below shows that the relative variation of the bag pressure $(d P / P)$ is linked to the relative variation of the mass $(d m / m)$ and to the relative variation of the volume $[d V / V ; \mathrm{Eq}$. (2)]. For a same range of bag pressure the size of the bag is linked to the volume and mass of air inside. These considerations show that with a big bag, a musician must create a larger variation of mass $d m$ through a larger insufflation $Q_{\text {in }}$, or a larger variation of volume $d V$ through a larger arm displacement $d y$ in order to generate the same variation of pressure $d P$ than with a smaller bag with the same inner pressure. In other terms, it means that it is "more economic" to produce a big variation of bag pressure with a small bag, whereas a big bag allows a more precise pressure control.

The bag's size also modifies the response of the bag felt by the musician's arm. Neglecting the variation of the mass, the bag responds to a displacement of the musician's arm by a variation of the pressure and therefore a variation of the reaction force. The bag can therefore be modeled as a simple spring characterized by its stiffness

$$
k=\frac{d F_{\mathrm{arm}}}{d y}=\frac{S_{\mathrm{arm}}^{2} P}{V} .
$$

If the contact surface $S_{\text {arm }}$ is similar, a bigger bag appears more compliant.

\section{EXPERIMENTAL SETUP}

The Galician bagpipe (gaita) used was built in 2003 by Cristobal Prieto. It is a professional model, suitable for traditional and more modern repertoire with a range of up to an octave and a fifth with the appropriate reeds. Although each maker has his own identity, this bagpipe is representative of a standardised version of Galician gaitas widely played in the region today. Although it still uses traditional cane reeds, the bagpipe is fitted with a synthetic bag made of GoreTex (Newark, DE) and covered with a velvet case. Its full volume, estimated by diameter measurements and conical reconstruction, is around 9 liters. The melodic pipe (chanter) uses a double reed and has a range extending from $B_{3}$ $(\approx 247 \mathrm{~Hz})$ to at least $E_{6}(\approx 1300 \mathrm{~Hz})$. The three drones, pitched at $C_{3}(\approx 130 \mathrm{~Hz}), C_{4}(\approx 260 \mathrm{~Hz})$, and $G_{4}(\approx 390 \mathrm{~Hz})$, all use traditional single reeds. During the experiments, the higher drone was blocked in order to insert the sensor allowing the inner bag pressure measurements (Sec. III A).

Like most Majorcan bagpipes, the one used for our experiment is tuned in $C$ sharp. In order to facilitate the comparison of the two instruments, they are assumed to have the same root note $(C)$, resulting in two different diapasons: $A_{4}$ $\approx 440 \mathrm{~Hz}$ for the Galician bagpipe and $A_{4} \approx 472 \mathrm{~Hz}$ for the Majorcan bagpipe. The Majorcan bagpipes (xeremies) used for the measurements was built by Xesus ("Chus") Solís based on the model patented by a duo of musicians known as the "Xeremiers de Sa Calatrava." It has a hybrid bag (a combination of synthetic and natural hide) manufactured by Bannatyne (West Lothian, Scotland, UK), covered with a cotton case. Its full estimated volume is around 15 liters. The melodic pipe is played with a cane double reed manufactured in Galicia by Brea (Arzúa, Galicia, Spain). Taking into account the adapted diapason, it has a range extending from $B_{2}(\approx 130 \mathrm{~Hz})$ to $C_{6}(\approx 1120 \mathrm{~Hz})$. The three drones, pitched at $C_{3}(\approx 140 \mathrm{~Hz}), C_{4}(\approx 280 \mathrm{~Hz})$, and $G_{4}$ $(\approx 420 \mathrm{~Hz})$, have single reeds made out of plastic (reeds Seipal made by Seivane, A Coruña, Galicia, Spain). Similarly to the Galician bagpipe, the higher drone was blocked during experiments in order to carry out the inner bag pressure measurements (Sec. III A).

The possible difference of the bag material properties between the two instrument is supposed to have a negligible influence on the control compared to the large difference of volume. All the elements of the instruments studied have been kept the same during all the experiments, reeds included.

\section{A. Measured parameters}

The air inflow $Q_{\text {in }}$ provided by the musician is measured directly on the instrument with a Hans Rudolph pneumotachometer (Shawnee, KS). The flow sensor is installed between the bag and the insufflation pipe. The bag pressure $P$ is measured by a pressure sensor connected to the inner bag through the opening of an unused drone.

As the measurement setup needs to be easily transportable, the arm movement is measured by a camera filming the back of the musician who has three markers stuck to his belt, his shoulder, and his elbow (black dots in Fig. 3). Tracking the trajectory of each marker allows us to estimate the angle 


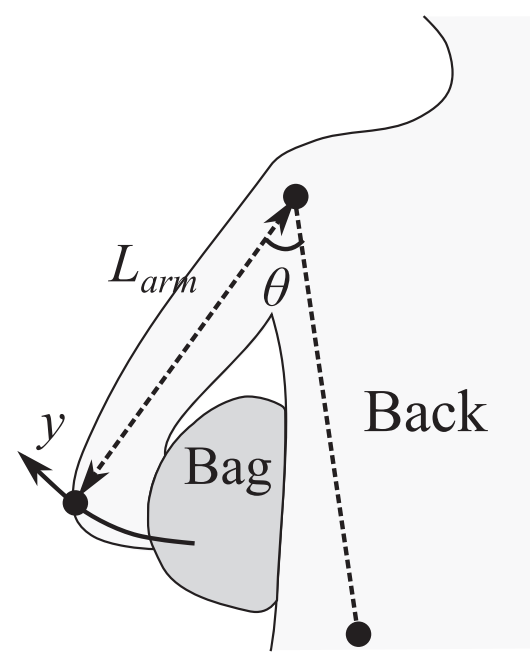

FIG. 3. Sketch of the motion capture setup.

$\theta$ between the arm and the body of the musician. Since the value of this angle is not absolute due to musicians' positions, the measurements presented are focused on the displacement of the arm, which is related to the variation of the angle

$$
d y=d \theta L_{\mathrm{arm}},
$$

with $L_{\text {arm }}$ the length of the upper arm of the musician. As illustrated in Sec. II, it is the sign of the displacement and its coordination with the insufflations that are essential for understanding the musician's control strategy.

Audio recordings are used to analyse the pitch variations of the notes, using the YIN pitch detection algorithm. ${ }^{11}$ Drone and melodic pipe frequencies are isolated, respectively, with a notch comb-filter and a peak comb-filter. In the results presented in this study, only the pitch accuracy of $C$ and $G$ are considered. Because the sounds of the pipes are simultaneously self-sustained, pitch accuracy is estimated in reference to pure intervals based on the mean frequency of the lowest drone $\left(f_{C_{5}}=4 f_{C_{3}}, f_{G_{5}}=6 f_{C_{3}}\right.$, etc. $)$.

\section{B. Acquiring data in a musical context}

For each bagpipe, three musicians with different levels of practice are recorded. Galician player "A" studied bagpipes episodically for 15 years and maintained an amateur practice. Galician piper " $\mathrm{B}$ " is a proficient player, winner of several local folk music contests with the traditional quartet formation. Galician player "C" has an international profile and is considered as one of the best gaita players of his generation. The first Majorcan player is an adult beginner, who has been playing for two years and the xeremies is his first instrument. The Majorcan player " $D$ " is a young player with 12 years of experience and is now considered as one of the next generation pipers, becoming a reference on the local scene as a piper. The Majorcan player " $E$ " is regarded as one of the best pipers on the island.

These musicians are asked to play several exercises: long steady notes (C5,G5,C6), long notes with an intended crescendo and decrescendo (C5,G5,C6), and finally diatonic scales that covered the entire range of the instrument. All these exercises are played only with the lowest drone $\left(C_{3}\right)$; the other drones are muted. The musicians also play an imposed musical piece. In Mallorca, they are all asked to play the "Bolero de Santa Maria," a 3/4 dance in $C$ major composed by Pep Toni Rubio in the 1980s and well known by bagpipe players of all levels. In Galicia, the imposed piece is "Loliña," a well-known 6/8 melody played in traditional environments. The Galician musicians are then asked to play a piece of their choice with pauses within the music as this is a musical effect used in this bagpipe culture. This is not required of Mallorcan pipers as their repertoire, whether traditional or more modern, does not use such stops within the music. During these musical pieces, the number of drones used is chosen by the musicians. Majorcan players use the two lowest drones $\left(C_{3}\right.$ and $\left.C_{4}\right)$ and the Galician musicians use only the lowest drone $\left(C_{3}\right)$.

\section{MECHANICAL CHARACTERISTICS OF THE INSTRUMENTS}

The aim of this section is to identify the global characteristics necessary to interpret the control of the instrument in musical context rather than characterize and model finely the behavior of the instruments. It is first important to identify the influence of the bag pressure on the pipes' behavior, especially in terms of pitch sensitivity and onset threshold. According to the model described in Sec. II, the value of the outflow $Q_{\text {out }}$ influences the control. An estimation of this outflow is therefore necessary to correctly interpret the control.

\section{A. Air consumption}

The air consumption of a bagpipe, which is a reed instrument, depends on several parameters. ${ }^{10}$ Some are fixed for a given instrument and piece of music such as the number of pipes used or the characteristics of the reeds. Others vary during the performance such as the inner bag pressure or the pitch of the notes played. Measuring the outflow during an instrumental performance in a noninvasive way is not easy. To accurately estimate this flow from the measurement of the pressure and the pitch it would be necessary to characterize the behavior of each pipe under controlled condition. Our setup allows for an estimation of the mean outflow.

During a long performance, excluding the beginning and the end, we suppose the average characteristics of the bag constant (pressure, volume, and mass of gas). This hypothesis implies that throughout the extract the mean outflow equals the mean inflow. Thus, the mean outflow can be estimated from the inflow measurement. To assure the comparability between the estimations, only long extracts $(>15 \mathrm{~s})$ using one drone and limited to the first register (under $C_{6}$ ) are taken into account. In total, five excerpts played by different players verified these hypotheses for the Galician bagpipe (corresponding to $283 \mathrm{~s}$ of signal) and six for the Majorcan bagpipe (corresponding to $261 \mathrm{~s}$ of signal).

The mean inflow extracted from these excerpts give an estimated outflow for the Galician bagpipe of $Q_{\text {out }}=5.0$ $\pm 0.2 \mathrm{cL} / \mathrm{s}$ and for the Majorcan bagpipe of $Q_{\text {out }}=7.5 \pm 2 \mathrm{cL} / \mathrm{s}$. The large variation obtained for the Majorcan bagpipe corresponds to a variation of values obtained by the different 
players. The Majorcan player D uses a particularly low air consumption compared to other Majorcan players (around $5 \mathrm{cL} / \mathrm{s}$ only). The bagpipe used for the experiments was his own so he may have learned how to optimize the control of his instrument in order to minimize the air consumption. Overall, however, the Majorcan xeremies seems to use more air than the Galician gaita. This difference should produce different control strategies for both instruments.

\section{B. Bag pressure range}

The inner bag pressure modifies several parameters of the sound produced by the instrument such as the pitch, the loudness, or the spectral content of the sound. Furthermore, the musicians must keep the pressure high enough to maintain a continuous sound. These different aspects impose the range of pressure in which the instrument can be played. This range can be different for each instrument. As illustrated in Sec. II, the value of the pressure influences the bag control.

The bag pressure is assumed to be independent of the number of pipes, but changes when the instrument is played in the second octave. Indeed, the pressure range within which the pipes sound is not modified by the number of pipe. However, the supply pressure is here the only control parameter, which allows the player to change the oscillating regime of the instrument (Fig. 4). In order to compare the musicians and the instruments, the bag pressure range is estimated on the musical excerpts ("Loliña" and "Santa Maria"), which have the same pitch range $\left(C_{5}-C_{6}\right)$. The pressure ranges used by the players are estimated by the 5 th and 95 th percentiles of the bag pressure, excluding the beginning and the end of the musical piece.

The ranges obtained for both instruments and all players are very similar, ranging from $5.1 \mathrm{kPa}$ to $6.4 \mathrm{kPa}$ for the Galician bagpipe and from $4.9 \mathrm{kPa}$ to $6.7 \mathrm{kPa}$ for the Majorcan bagpipe. This range is similar to the one already observed on Scottish bagpipes ([6 kPa,7.5 kPa]). ${ }^{7}$

\section{Pitch sensitivity}

The modification of the pitch by the bag pressure is a well-known effect by pipers, particularly for the drones. The
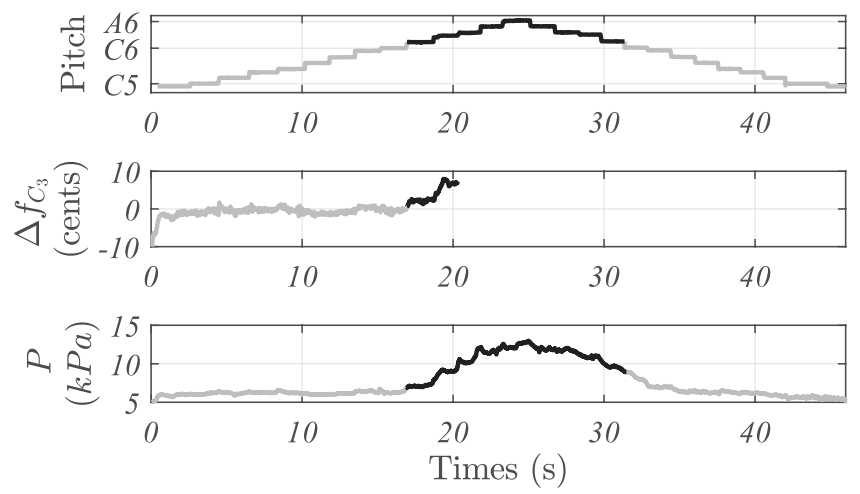

FIG. 4. Parameters during a scale over 1,5 octave by the Galician player C: the pitch of the chanter in logarithmic scale (pitch), the pitch variation of the drone around its mean frequency $\left(\Delta f_{C 3}\right)$, and the bag pressure $(P)$. The gray lines correspond to the first octave and the black lines to the second octave. The drone stops during the second octave due to the high pressure of the bag (Sec. IV D). stability of the drone pitch during a performance is one criteria to evaluate the level of expertise of a player. ${ }^{12}$ The bag pressure also modifies the loudness and the spectral content of the sound. ${ }^{6}$

The sensitivity of the pitch in relation to the bag pressure is measured from the crescendo-decrescendo notes. During this exercise the players were asked to vary the loudness of three notes $\left(C_{5}, G_{5}, C_{6}\right)$ as much as possible without paying attention to pitch. This led them to explore a much larger range of bag pressure than the one used during the musical pieces. Besides the crescendo note, the data of the drone pitch from all the recordings contributes to the estimate the drone sensitivity.

The data obtained for the evolution of pitch in relation to the bag pressure (Fig. 5) are consistent with the observations made under controlled conditions. ${ }^{6}$ Using linear regression in the range of $5 \mathrm{kPa}<P<6.5 \mathrm{kPa}$, it is possible to extract for each player the sensitivity of the drone $\left(C_{3}\right)$ and the sensitivity of the melodic pipe for the notes played $\left(C_{5}, G_{5}, C_{6}\right.$; Fig. 5). The overall offset, which can be different for each musician, indicates that the instruments were tuned differently by each of them. The slope is therefore estimated independently for each musician.

The treatment of the data for all the players give a global sensitivity around 4 cents/ $\mathrm{kPa}$ for the Galician drone and 15 cents $/ \mathrm{kPa}$ for the Majorcan drone. As the properties of the melodic pipe are modified by the different fingerings used for each note, the three chanter's notes studied here $\left(C_{5}, G_{5}, C_{6}\right)$ present different sensitivity rates (Fig. 5). It is, for example, visible with the Galician piper "B" for which the estimated sensitivity of $G_{5}$ is around twice the sensitivity of the $C_{5}$ and $C_{6}$ (Fig. 5). In spite of this intra-variability, the sensitivities are more or less independent of the player for a given note and bagpipe. To summarize this comparison, the mean sensitivity of the chanter is defined for each musician by taking the average of the sensitivities of the three notes studied. Finally, the mean chanter sensitivity is around 10 cents $/ \mathrm{kPa}$ for the Galician bagpipe and around 20 cents $/ \mathrm{kPa}$ for the Majorcan bagpipe (Fig. 5). These mean values are about twice as high for the Majorcan chanter as for the Galician one. The pitch of the Majorcan bagpipe is therefore much more sensitive to pressure variations than the Galician one for both the drone and the chanter (melodic pipe).

Knowing the differences in pitch sensitivity, the similarities between the range of bag pressure used for the two instruments $(5 \mathrm{kPa}<P<6.5 \mathrm{kPa}$, Sec. IV B) seem to indicate that pitch accuracy does not impose a strong restriction in the pressure range used. Indeed, the pitch of the drone varies about 30 cents if a Majorcan player explores the full range of pressure, whereas the pitch of the Galician drone varies only about 7 cents for the same range of pressure. The origin of this limited range may be the range in which the melodic pipe and the drones have similar sensitivities, which allows the musician to maintain a melody in tune with the drones when the bag pressure varies. ${ }^{6}$ Additionally, the low boundary of the range may be determined by the offset threshold of the pipes: the value of the bag pressure under which the sound stops, which is explored in Sec. IV D. 


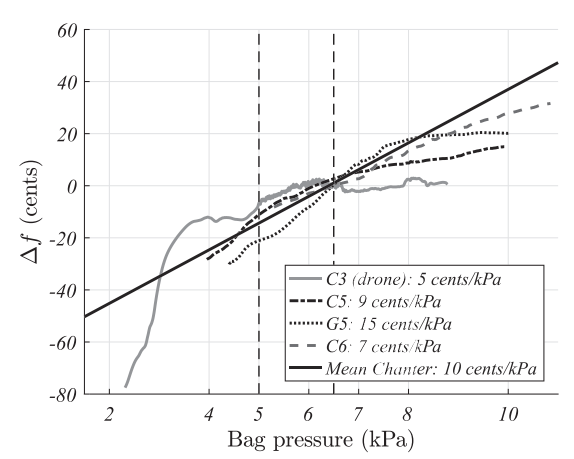

(a)

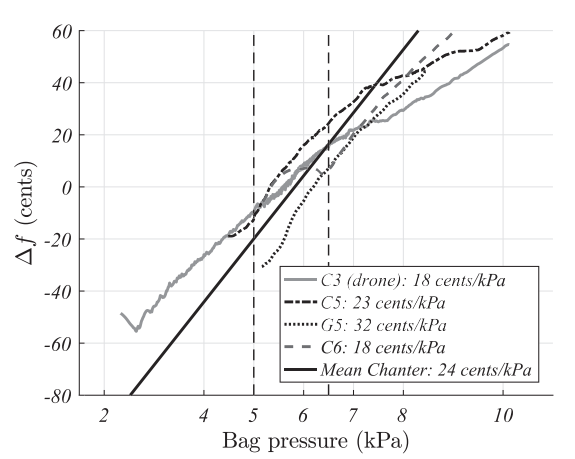

(b)

FIG. 5. Pitch sensitivity with the bag pressure estimated by linear regression in the range of interest indicated by vertical dashed lines $(5 \mathrm{kPa}<P<6.5 \mathrm{kPa})$. They are estimated from the data of the crescendo exercise for the melodic pipe $\left(C_{5}, G_{5}, C_{6}\right)$ and global data for the drone $\left(C_{3}\right)$. The frequency reference for the drone has been fixed to $131 \mathrm{~Hz}$ for the Galician bagpipe and $140 \mathrm{~Hz}$ for the Majorcan one. The reference values for the chanters' notes are calculated in order to create pure intervals with the drones. The mean melodic pipe sensitivity is estimated from the average sensitivity of the three notes studied. (a) Galician player "B." (b) Majorcan player E.

\section{Onset and offset thresholds of the bagpipe}

In a musical context the onset and offset thresholds are relevant at the beginning and the end of the musical piece. They also give the musician the possibility to create pauses during the performance. The bag pressure for which the oscillation starts or stops is influenced by the dynamic of the bag pressure evolution: the faster the evolution, the more the threshold shifts. ${ }^{13}$ For the onset, the faster the increase of pressure, the higher the pressure when the oscillation starts.

Due to the context in which the measurements were carried out, only an upper estimation of the pipes' onset threshold can therefore be provided by taking the minimal onset pressure observed.

These onsets are estimated from the observation of the pressure profile at the beginning of the piece (Fig. 6). For both bagpipes, the melodic pipe has an onset threshold of around $5 \mathrm{kPa}$. The minimal value at which the Galician drone starts is around $4 \mathrm{kPa}$ (player C, Fig. 6). By adding the fact that the drone does not start when the bag pressure is maintained around $3 \mathrm{kPa}$ (player A, Fig. 6) suggest that the onset threshold of the Galician drone is ranged between $3 \mathrm{kPa}$ and $4 \mathrm{kPa}$. In a similar way, the pressure profile of the Majorcan player E seems to indicate that the Majorcan drone's onset threshold is under $1 \mathrm{kPa}$ (Fig. 6, bottom).

For the Galician bagpipe, the onset thresholds of the pipes are similar enough to allow the simultaneous onset of the chanter and drones by using the dynamic behavior of the instrument (players "A" and "B," Fig. 6, top). The fast increase of the bag pressure is furthermore facilitated by the small volume of the bag (Sec. II). On the other hand, the low onset threshold of the Majorcan drone and the large volume of the bag makes this kind of start difficult. Furthermore, the bag empties itself mainly through the drone when the musician tries to fill it due to its volume and the amount of time needed to fill it with air. These two difficulties are well known by the players. In order to limit these effects, the musicians block the entry of the drones with their fingers from the outside of the bag when they are filling it with air. Here, this strategy is used by player D. By increasing the pressure rapidly, he is able to reach the high onset threshold observed for the drone (Fig. 6, bottom).

For the Galician bagpipe, a lower cutoff value of the offset thresholds can be estimated with the pauses within a musical piece. It appears that the melodic pipe stops if the bag pressure dips under $4 \mathrm{kPa}$ and the drone stops when the bag pressure dips under $2 \mathrm{kPa}$ (Fig. 11). Furthermore, the offset threshold for a given pipe must be lower than the onset threshold by definition. The offset of the Galician melodic pipe is therefore between $4 \mathrm{kPa}$ and $5 \mathrm{kPa}$ and that of the drone is between $2 \mathrm{kPa}$ and $4 \mathrm{kPa}$. The offset of the Majorcan drone is very low (under $1 \mathrm{kPa}$ ), but cannot be more precisely estimated from the measurements presented. The offset of the Majorcan chanter can be approximated by observing the crescendo-decrescendo notes (Fig. 5), or the control of the beginner who is unsuccessful in maintaining a continuous sound (Fig. 7). It seems to be around $4 \mathrm{kPa}$.

From these consideration, the lower boundary of the bag pressure range $(\approx 5 \mathrm{kPa})$ seems to be linked to the offset threshold of the chanter. Aside from the consequences on the control strategies, the difference of the drone onset thresholds between both instruments influences musical aspects. For example, the ability of Galician pipers to play musical pieces with pauses is partly facilitated by the high values of the onset and offset thresholds for both the melodic pipe and the drones.
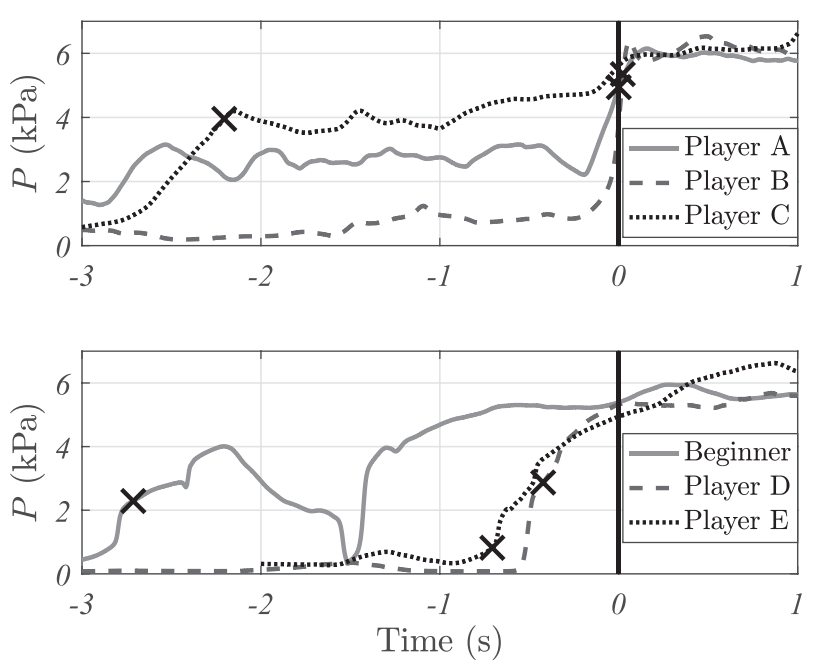

FIG. 6. Comparison of onset of the instrument for different players for the Galician (top) and the Majorcan bagpipes (bottom). The signals are synchronized on the onset of the melodic pipe fixed arbitrarily at $0 \mathrm{~s}$ (vertical black line). The drone onsets are indicated by a black cross. 
For single-reed and double-reed instruments, an upper boundary of pressure exists over which the reeds are blocked and the instrument stops playing.9,10 This boundary could explain the upper limits of the range used by the players. For the Galician drone, this value is attained by player $\mathrm{C}$ during the diatonic scale (Fig. 4). The drone seems to stop over $9 \mathrm{kPa}$. This threshold is not attained by the Majorcan players.

\section{E. Summarizing the comparisons}

The characteristics of the two instruments studied are summarized in Table I. The behaviors of the instruments being sensitive to many manufacturing aspects, Table I is $a$ priori not generalizable to all gaita and xeremies.

\section{FINE CONTROL OF THE BAG PRESSURE}

The behavior of the instrument is now described, and the aim is to study the control of the bag pressure during the musician's performance. In order to identify the different ways to control the bag pressure for both instruments, the steady notes are first observed. Second, the study of the bag's pressure variations during musical performances allows us to determine if they are led by the music.

\section{A. Steady sound}

Musicians were asked to play slow arpeggio $\left(C_{5}, G_{5}\right.$, $\left.C_{6}, G_{5}, C_{5}\right)$ and to keep a steady sound. For this to happen, the musicians were expected to keep the bag pressure more or less constant during a given note. These measurements allowed us to observe the acceptable order of magnitude of bag pressure in order to produce a "steady" sound and which elements of control are used by the players. Indeed, according to the model in Sec. II, different controls can lead to a
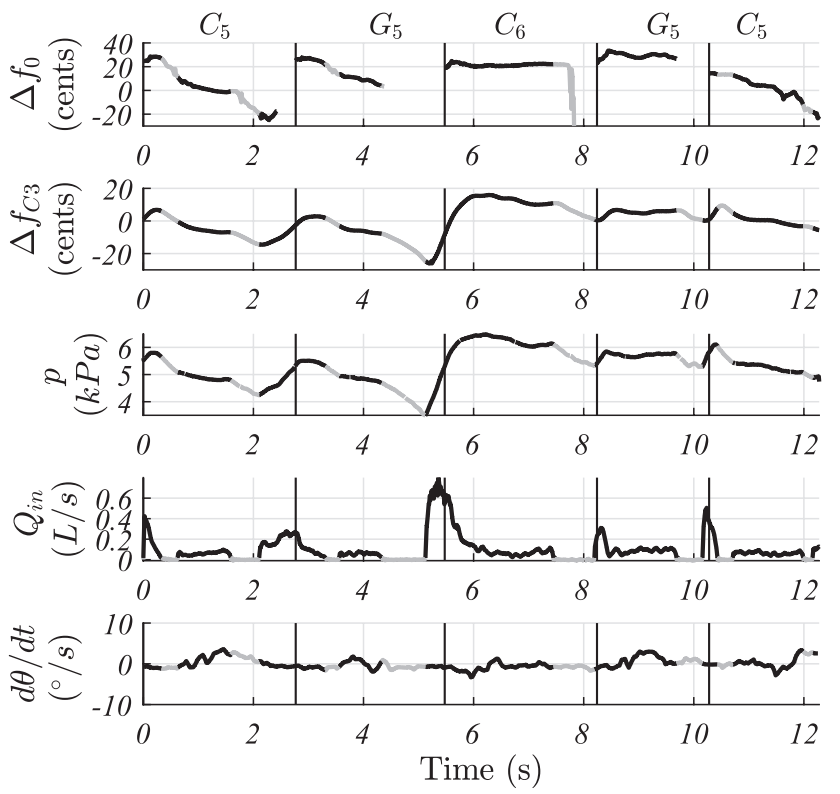

FIG. 7. Control parameters of the Majorcan beginner during steady notes: the pitch variation of the melodic pipe $\left(\Delta f_{0}\right)$, the pitch variation of the drone around its mean frequency $\left(\Delta f_{C 3}\right)$, the bag pressure $(P)$, the flow injected by the musician $\left(Q_{\text {in }}\right)$, and the angular velocity of the arm $(d \theta / d t)$. The gray parts of the lines correspond to the instant when the musician does not blow into the bag $\left(Q_{\text {in }} \approx 0\right)$.
TABLE I. Global characteristics of the bagpipes studied.

\begin{tabular}{|c|c|c|}
\hline Bagpipe & Galician & Majorcan \\
\hline Diapason & $A_{4} \approx 440 \mathrm{~Hz}$ & $A_{4} \approx 472 \mathrm{~Hz}$ \\
\hline Bag Volume & $V \approx 9 \mathrm{~L}$ & $V \approx 15 \mathrm{~L}$ \\
\hline Chanter pitch & {$\left[B_{4}, E_{6}\right]$} & {$\left[B_{4}, C_{6}\right]$} \\
\hline Chanter reeds & \multicolumn{2}{|c|}{ Double cane reed } \\
\hline Drones pitch & \multicolumn{2}{|c|}{$C_{3}, C_{4}\left(G_{4}\right.$ unplayed $)$} \\
\hline Drones reeds & Single, cane & Single, plastic \\
\hline Chanter onset & \multicolumn{2}{|c|}{$\approx 5 \mathrm{kPa}$} \\
\hline Chanter offset & \multicolumn{2}{|c|}{$\approx 4 \mathrm{kPa}$} \\
\hline Drone onset & $\approx 4 \mathrm{kPa}$ & $\approx 1 \mathrm{kPa}$ \\
\hline Drone offset & $\approx 2 \mathrm{kPa}$ & $<1 \mathrm{kPa}$ \\
\hline Pressure range & \multicolumn{2}{|c|}{$[5,6.5](\mathrm{kPa})$} \\
\hline Chanter Sensitivity & $\approx 9 \mathrm{cts} / \mathrm{kPa}$ & $\approx 17 \mathrm{cts} / \mathrm{kPa}$ \\
\hline Drone Sensitivity & $\approx 4 \mathrm{cts} / \mathrm{kPa}$ & $\approx 15 \mathrm{cts} / \mathrm{kPa}$ \\
\hline Air consumption & $\approx 5 \mathrm{cL} / \mathrm{s}$ & $\approx 7.5 \mathrm{cL} / \mathrm{s}$ \\
\hline
\end{tabular}

variation of the bag pressure: the variation of strength linked to a variation of the arm displacement or the delay between insufflations and arm displacements, which are particularly visible at the beginning and the end of the insufflations.

The different parameters measured and their exploitation allows the simultaneous observation of the pitch of the melodic pipe $\left(\Delta f_{0}\right)$ and the drone $\left(\Delta f_{C 3}\right)$, which are expressed here in cents in reference to pure intervals based on the mean drone frequency (Sec. III A), the bag pressure $(P)$, the inflow $\left(Q_{\text {in }}\right)$, and the arm displacement associated with the angular velocity $(d \theta / d t \propto d y / d t$, Sec. II; Figs. 7 and 8). In order to highlight the coordination between the arm displacement and the insufflations, the data are plotted in gray when the inflow is null and in black when is it positive.

During this exercise, it appears that the beginner does not move his arm (Fig. 7). The player used one insufflation per note. Here, the bag is not used as an air reservoir, which leads to a large variation of the bag pressure at each insufflation. These variations also produced a large variation of the drone pitch and were large enough to stop the sound of the melodic pipe. The beginner cannot maintain enough high pressure to produce a continuous sound without a good synchronization between insufflations and arm displacements.

The other players have enough control to ensure a continuous sound (Fig. 8). The variation of the bag pressure for one given note consistently remained under $0.5 \mathrm{kPa}$. For the Galician players [Figs. 8(a) and 8(b)], the coordination between the inflow and the arm displacement is particularly visible: when the player blows into the instrument, his arm goes up $(d \theta / d t>0)$ and inversely. No pattern within the bag pressure is synchronized with the insufflations which underlines a good coordination. The weak pitch sensitivities of the pipes smooth the small fluctuations of the bag pressure and allows the musician to produce steady pitch both with the melodic pipe and the drone: the pitch variations are less than 5 cents for any given note on both the drone and melodic pipe for both players [Figs. 8(a) and 8(b)]. Player A also maintains the bag pressure constant for the arpeggio. With this pressure profile, the $G_{5}$ then the $C_{6}$ are slightly too flat with respect to the drone: the intervals are not pure [Fig. 8(a)]. Player $\mathrm{C}$ corrects this aspect by increasing the bag pressure by around 

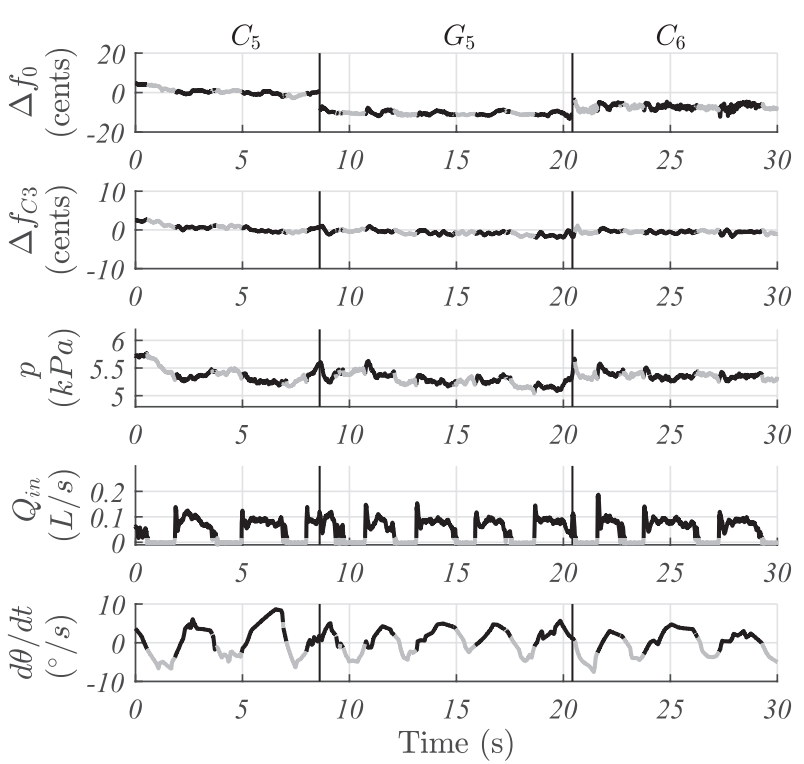

(a)
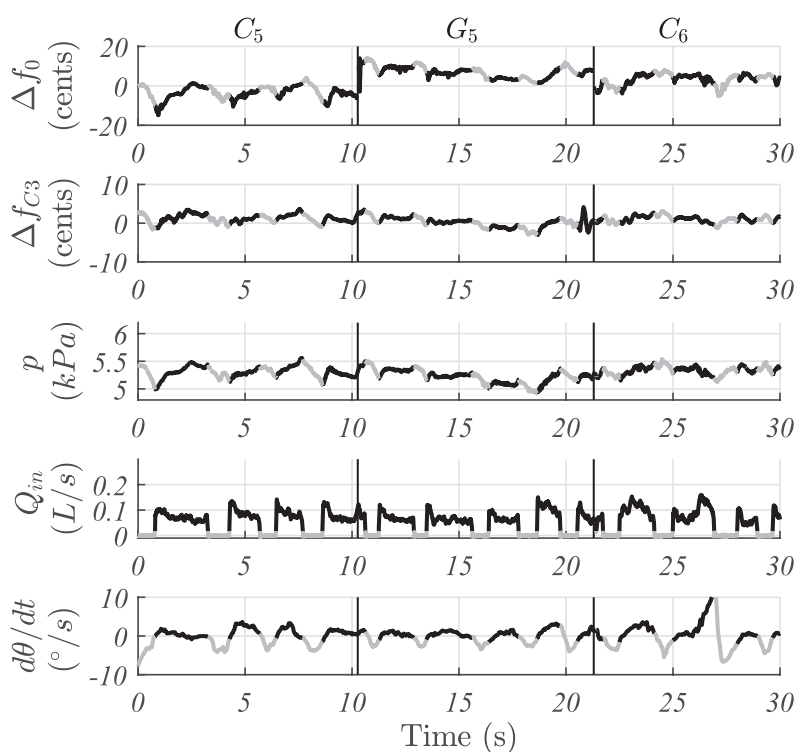

(c)
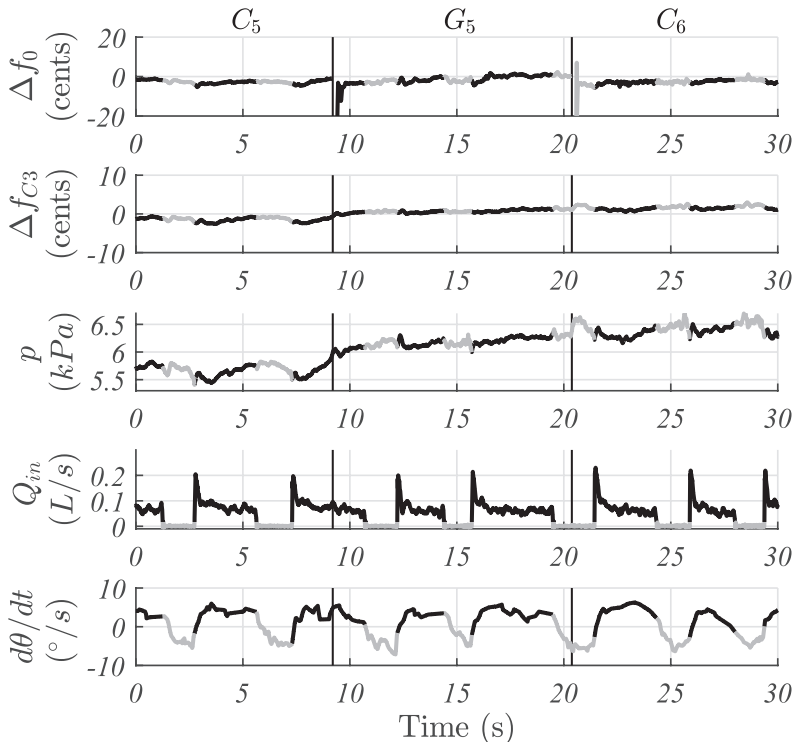

(b)
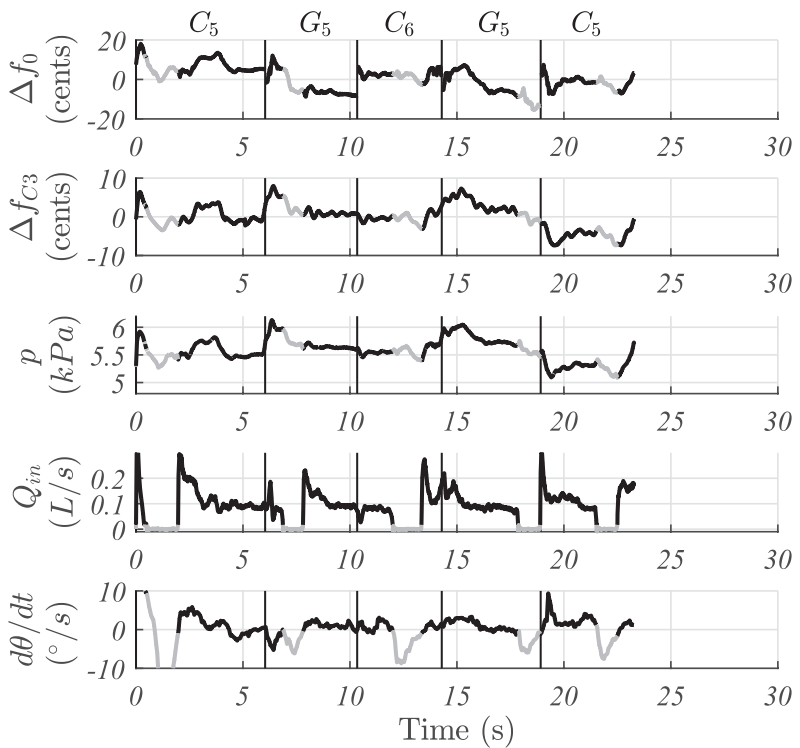

(d)

FIG. 8. Control parameters during steady notes: the pitch variation of the melodic pipe $\left(\Delta f_{0}\right)$, the pitch variation of the drone around its mean frequency $\left(\Delta f_{C 3}\right)$, the bag pressure $(P)$, the flow injected by the musician $\left(Q_{\text {in }}\right)$, and the angular velocity of the arm $(d \theta / d t \propto d y / d t$, Sec. II). For the comparison, the same temporal scale is kept between the musician leading to a different number of visible notes. The gray parts of the lines correspond to the instant when the musicians do not blow into the bag $\left(Q_{\text {in }}=0\right)$. (a) Galician player A. (b) Galician player C. (c) Majorcan player D. (d) Majorcan player E.

$0.5 \mathrm{kPa}$. As the pitch of the chanter is more sensitive to the bag pressure than to the pitch of the drone (Sec. IV C), the variation of the bag pressure allows the musician to play the melodic pipe in tune with the drone [Fig. 8(b)].

With the Majorcan players, the coordination between the arm and the insufflation is less visible due to a smaller variation of the detected arm speed [Figs. 8(c) and 8(d)]. For this instrument, both arms embrace and compress the bag and additionally to the side measurement measured here, their movement also includes a frontal displacement. The angle $\theta$ represents, therefore, less accurately the compression of the bag. In spite of this, the quality of the insufflation-arm coordination can be evaluated from the variation of the bag pressure. For the Majorcan player D, the fluctuation of the bag pressure appears to be coordinated with the blowing: the bag pressure increases during the insufflations (black) and otherwise decreases [gray; Fig. 8(c)]. These fluctuations could be linked to an arm displacement that is too small and therefore does not compensate the insufflations. For player E, the variation of the bag pressure is larger but does not seem to be coordinated with the blowing [Fig. 8(d)]. More specifically, this player seems to accentuate the beginning of some notes (especially $G_{5}$ ). In this instance, the pressure increase seems to be controlled, such as at the beginning of the first $G_{5}$ (at around $6 \mathrm{~s}$ ) when the player starts to compress the bag before the end of the insufflation [Fig. 8(d)]. Due to the high pitch sensitivity of this instrument, the fluctuation of the bag pressure creates a wide fluctuation of pitch. For example, the poor control of the bag pressure by player D induces a quasi-regular fluctuation of the chanter pitch about 10 cents all along the exercise. 
The accentuation of the $G_{5}$ by Majorcan piper "E" and the correction of pitch through the variation of the bag's pressure by Galician player $\mathrm{C}$ suggest that the bag pressure can be varied intentionally by the player in response to the musical task.

\section{B. Pressure variations in a musical context}

To study the link between the bag pressure fluctuation and the musical task, it is interesting to compare the bag pressure profile between two repetitions of the same task for the same player but also to compare this profile between two musicians playing the same exerpt. If similar pressure profiles are observed for two repetitions by the same musician, it will suggest that the player controls the fluctuation in response to the musical task. As evoked in Sec. V A, the fluctuation could be induced by musical intent (accent, crescendo, etc.) or to correct the pitch. The comparison of the pressure profile between two players can help to distinguish these two types of control. As presented in Sec. II, the player has two main parameters to control the bag pressure: the insufflation and the arm displacement. The comparison between players also has the aim to identify different control strategies that can be used. In a traditional music context, the instrument is associated to a specific repertoire. A different musical piece was therefore studied for each of the two instruments.

For bagpipes, like many drone instruments, pitch is tuned relatively to the drone with pure fourths, fifths, and octaves. The intermediate intervals, traditionally less important in the melodic structure, tend to fluctuate more.

\section{Galician players}

In Galician traditional music the phrases are often repeated twice. This is ideal to observe the link between the bag pressure profile and the musical task. Here, the second phrase of "Lolina" is analysed. The score is given on Fig. 9(a).

For both players, the bag pressures vary on a range of $1 \mathrm{kPa}$ along the repeated phrase (Fig. 9). For each of the two Galician players observed, the bag pressure profiles are similar for both occurrences [Figs. 9(b) and 9(c)]. For Galician player " $B$ " the profiles are specifically characterized by marked local maxima of bag pressure coordinated with each repetition of the triplets $E, D, C$ (bars 1 and 5 with their upbeats) and a smoother evolution for the rest of the phrases [Fig. 9(b)]. The peaks observed on the bag pressure suggest that this musician wants to accentuate this pattern. The pressure profile used by musician $\mathrm{C}$ does not accentuate the first beat of the descending $E, D, C$ quavers [Fig. 9(c)]. Musician $\mathrm{C}$ seems to give an overall smooth shape to the bag pressure for each group of four bars. This analysis shows that the player can finely control the bag pressure of the gaita and that the bag pressure profile depends on the musician's interpretation of the musical task.

The variations of the bag pressure induced fluctuations of the drone pitch. The magnitude of these fluctuations stays under five cents for both players [Figs. 9(b) and 9(c)] in spite of a bag pressure range of about $1 \mathrm{kPa}$. This difference is enabled by the low pitch sensitivity of the instrument (Sec. IV C). The pitch variations are smaller for player C than B despite having same order of magnitude of pressure variations (Fig. 9). This difference could be associated to the higher range of pressure used by player $\mathrm{C}$ inducing some nonlinearities in the drone behavior.

To control the variations of the bag pressure, the musicians do not need to synchronize their overall control. Indeed, both musicians obtained similar bag pressure profiles

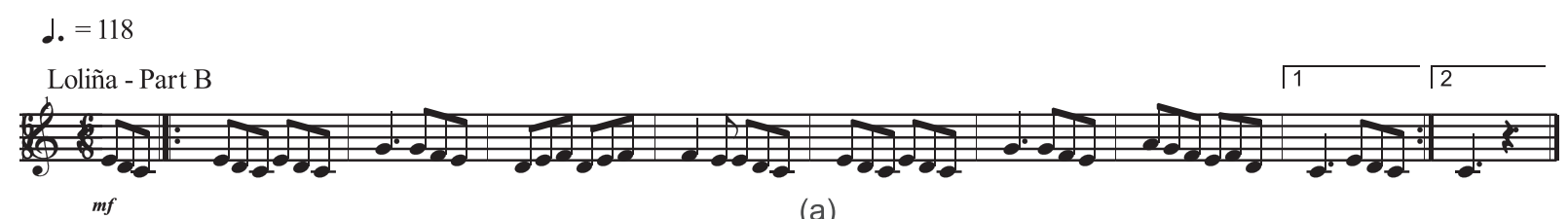

(a)
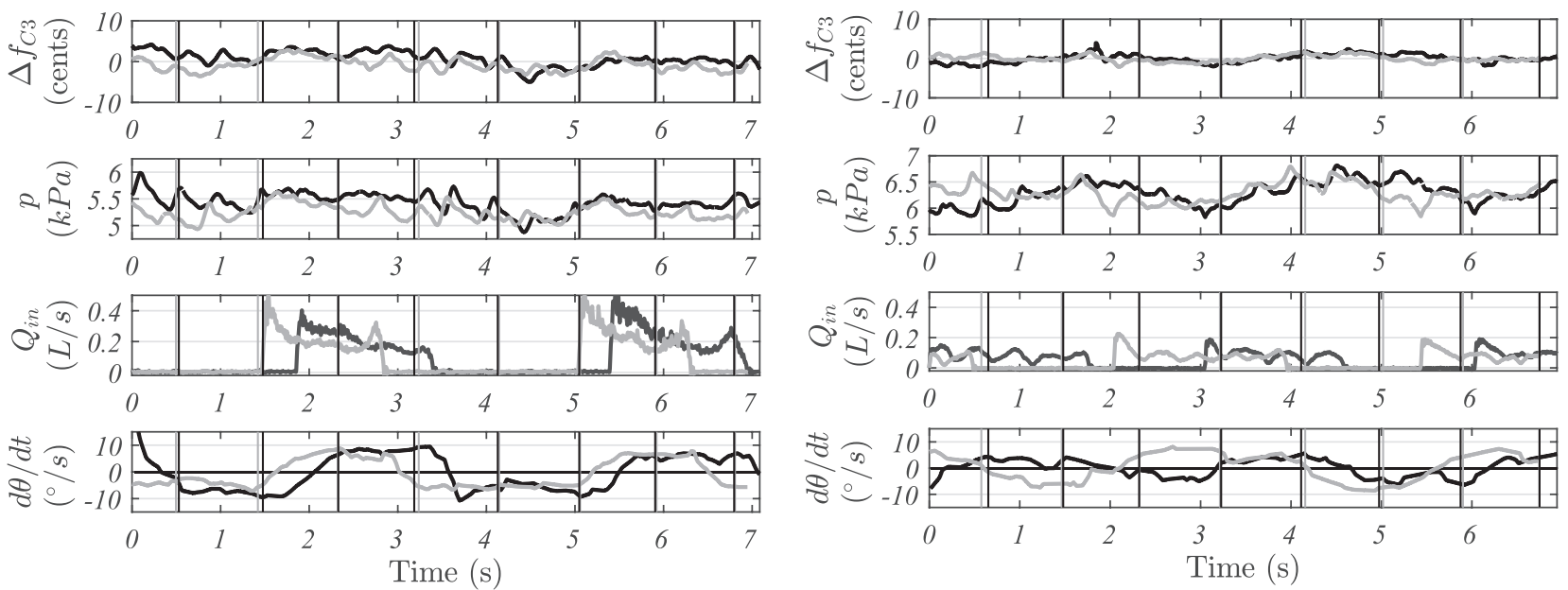

(b)

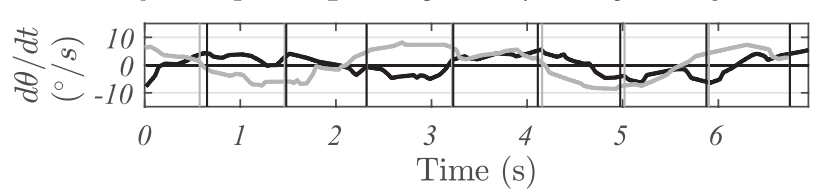

(c)

FIG. 9. Repeatability of the bag pressure control during a traditional Galician piece of music [score on (a)]. The black lines correspond to the first occurrence of the musical sentence and the gray line to the second occurrence. For each occurrence, the vertical lines represent the barlines of the musical score. For each graph, the uppermost plot is the pitch variation of the drone $\left(\Delta f_{C 3}\right)$ around its mean frequency, the second is the bag pressure $P$, the third is the flow $Q_{\text {in }}$ injected by the musician, and the last is the angular velocity of the arm ( $d \theta / d t \propto d y / d t$, Sec. II). (a) Score. (b) Galician player "B." (c) Galician player C. 
with very different control patterns (insufflation and arm displacements; Fig. 9). The musicians kept a regular insufflation rhythm, coordinated with the arm displacement all along the phrase. The rhythm and the durations of all insufflations stayed the same for the length of the excerpt. To the control necessary for the proper use of the instrument, an extra layer of fine control is added. The latter small arm displacements, which allow the musician to provide the small bag pressure variations described previously, are not particularly visible on the measurements due to the poor accuracy of the setup used (Sec. III A).

Although the musical pressure variations and the overall air supply of the instrument are not synchronized, some unforeseen fluctuations of the bag pressure can be linked to a coordination delay between the arm displacements and the insufflations. For example, a small decrease in the bag pressure appears at the beginning of each insufflation for player $\mathrm{C}$ that is not synchronized with the musical task and is therefore thought to be unintentional [Fig. 9(c)].

\section{Majorcan players}

In the Majorcan bagpipe tradition, musical pieces are generally preceded by an improvised prelude, which has the function of stabilization of the reeds and tune melodic pipe to the drones. ${ }^{14}$ For this comparative study, the preludes had to be excluded. Instead, we used the second phrase of "Bolero de Santa Maria" composed by Pep Toni Rubio. The corresponding score is given in Fig. 10(a). Due to the sequence of the musical phrases, the beginning and the end of the excerpts studied are slightly different for each occurrence.

The bag pressure ranges used by the two players studied here have a width around $1 \mathrm{kPa}$ (Fig. 10), similar to the range used by the Galician players. Here again, for each player, the bag pressure profiles are similar for the two occurrences [Figs. 10(b) and 10(c)]. For the Majorcan player E, the profiles differ during the first and last bars due to the sequence of the musical phrases. Aside from these bars, the profiles have a similar global shape for both players even if musician $\mathrm{E}$ marks more the variation [Figs. 10(b) and 10(c)]. It is therefore more difficult to determine if the bag pressure variations are used to tune the instrument or to modulate the sound following an expressive intention. The bag pressure evolves during the steady note and shows similar profiles for each bar, which seems to indicate that the bag pressure varies here according to an expressive intention. The profile is particularly identifiable for player E [Fig. 10(c)]: for each bar the bag pressure increases during the steady notes, is maximal for the sixteenth notes then decreases until the end of the bar.

If we compare these to the bag pressure profiles observed for the Galician bagpipe (Fig. 9), the Majorcan variations seem to be slower and smoother (Fig. 10). These differences could be linked to the bag size. Indeed, as evoked in Sec. II, for a similar mean bag pressure, a larger displacement is necessary to induce the same variation of bag pressure with a big bag than with a smaller one. It is therefore easier to carry out quick variations of the bag pressure with the Galician bagpipe than with the Majorcan one (Table I).

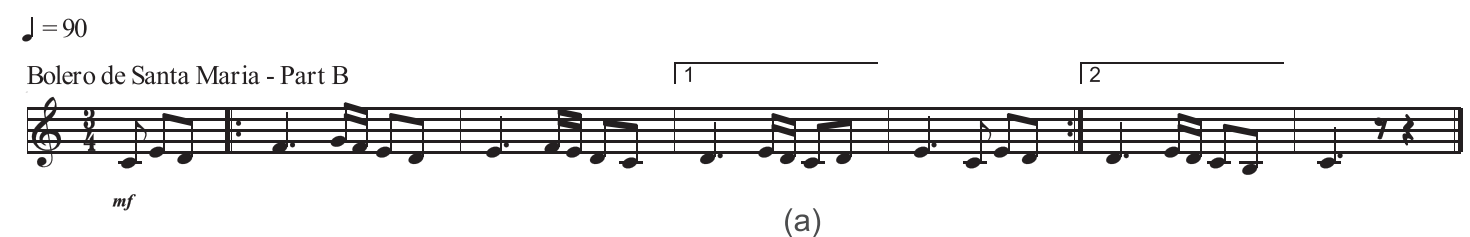

(a)
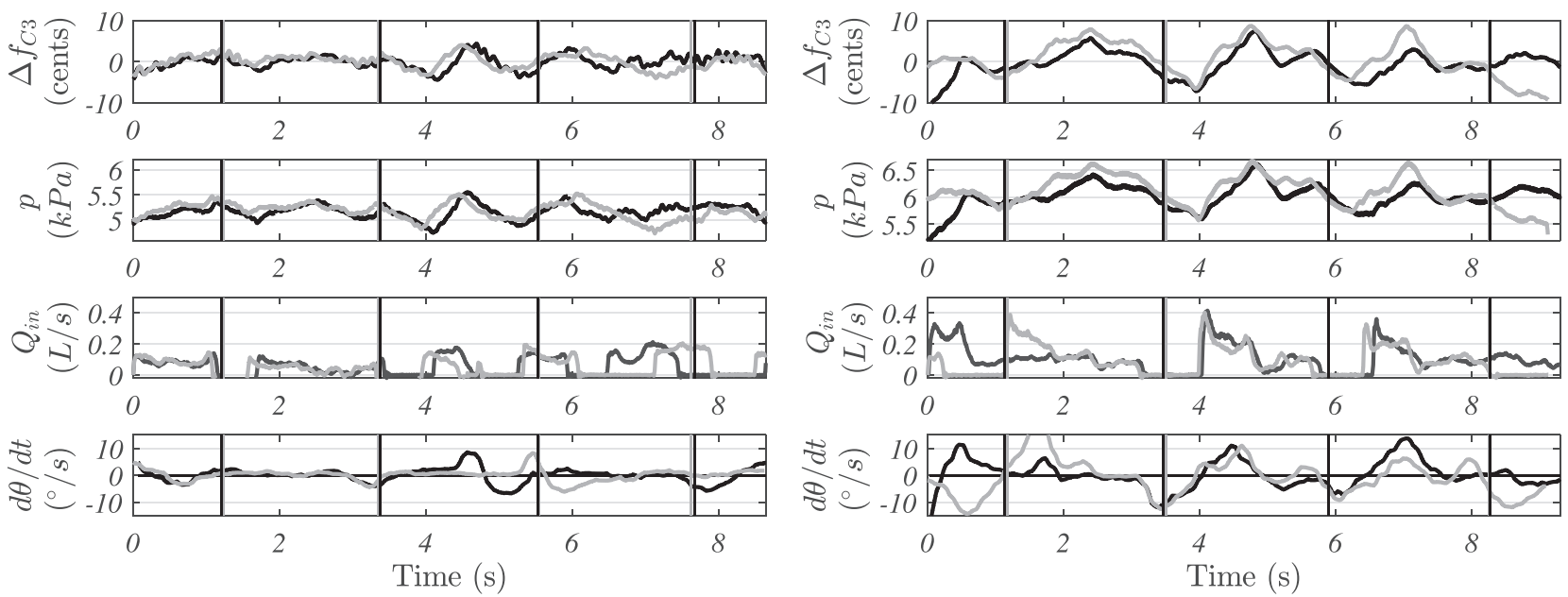

(b)

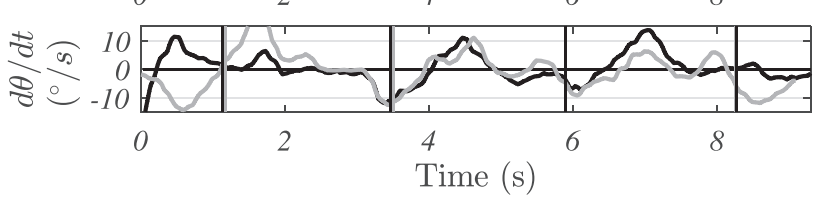

(c)

FIG. 10. Repeatability of the bag pressure control during a traditional Majorcan piece of music [score on (a)]. The black lines correspond to the first occurrence of the musical sentence and the gray line to the second occurrence. The vertical lines represent the bars of the musical score. For each graph, the uppermost plot is the pitch variation of the lowest drone $\left(\Delta f_{C 3}\right)$ around its mean frequency, the second is the bag pressure $P$, the third is the flow $Q_{\text {in }}$ injected by the musician, and the last is the angular velocity of the arm $\left(d \theta / d t \propto d y / d t\right.$, Sec. II). For this music piece, the Majorcan players used two drones $\left(C_{3}\right.$ and $\left.C_{4}\right)$. (a) Score. (b) Majorcan player D. (c) Majorcan player E. 
The difference of tempi and rhythm (slower in the Majorcan tune) could also explain, in part, these differences.

The differences of the bag pressure induce a relatively important variation of the drone pitch with a magnitude of around 20 cents for the Majorcan player E [Fig. 10(c)]. This variation is large enough to be audibly perceptible. It seems that in this context, musicians choose to privilege musical expression over pitch stability.

To obtain these bag pressure profiles, it appears here that the musicians synchronize the bag control with the musical task. Indeed, as we can read on the measurements, both the insufflation and the arm displacements are repeated in the same way for both occurrences and for the two musicians studied [Figs. 10(b) and 10(c)]. For both musicians, the rhythm of insufflation is irregular, contrary to what was observed during the steady notes with the same players (Figs. 8(c) and 8(d)). These irregularities are particularly visible with the Majorcan player D who alternates long and weak insufflations with shorter and more intense ones [Fig. 10(b)]. For both musicians, this rhythm is repeated for the two occurrences and synchronized with the musical task, except for the first and last bars that, as we pointed out earlier, change according to the musical sequence. For example, player E seems to coordinate his insufflations with the increase of the bag pressure. The timing and shape of the insufflation is also maintained for both occurrences in particular by player E who repeats it almost exactly [Fig. 10(c)]. The synchronization of the control gesture with the musical task is also visible on the arm displacement. This repeatability is particularly visible with the player E for whom the variation of angle has a bigger magnitude [Fig. 10(c)].

For this instrument, the musicians modify the coordination between insufflation and arm displacement in order to control the variation of the bag pressure. This desynchronization is particularly visible with the Majorcan player D who sometimes decreases the bag's volume $(d \theta / d t<0)$ while he is still inflating it $\left(Q_{\text {in }}>0\right)$. This movement is used just before the first and the second bars and just after the third one [Fig. 10(b)]. This control gesture allows him to increase the bag pressure. The same kind of control is used by player E who sometimes stops moving his arm during the insufflation in order to obtain a high pressure [at the middle of each bar Fig. 10(c)].

Different control strategies are observed between the Galician and the Majorcan players: the Galician players maintain a regular insufflation and arm displacement rhythm. whereas the Majorcan players adapt their control gesture to the musical task. This difference could be linked to the size of the bag. Due to the large volume of the bag, the players have to create a bigger displacement or insufflation in order to produce the same pressure variation on the Majorcan bagpipe as on the Galician bagpipe (Sec. II). By combining insufflation and arm displacement, the Majorcan players can attain the same range of bag pressure variation. This difference of control also allows us to interpret the slower variation observed on the Majorcan bagpipe; the bag pressure is less sensitive to small variations which might explain why the bag pressure profiles are smoother for the Majorcan bagpipe than for the Galician bagpipe.

\section{Strategy in a specific context: Pauses}

The small size of the gaita's bag and the sensitivity of the bag pressure to the associated control gesture allows the player to produce large and sharp variations of the bag pressure, which can be used in a musical context. In these specific contexts, the desynchronization of the insufflation and global arm displacement with the musical task is no more valid.

Some Galician pieces include pauses during which both the drones and melodic pipe stop for a short period. To create these pauses, the players must decrease the bag pressure sufficiently in order to reach the offset of all the pipes and then increase the pressure to start the sound again. This large variation of the bag pressure must be perfectly controlled and quickly executed in order to respond to the musical task. This effect is therefore associated to a specific control of the bag. Indeed, the bag must be empty enough in order to allow the musician to abruptly and sufficiently reduce the bag pressure.

The Galician musician B played the traditional piece "Roxelio" in which one phrase contained a succession of two pauses (Fig. 11). This phrase appears twice in the musical piece and is repeated each time, which gives four occurrences of this excerpt by the same musician. In this specific context, besides the repeatability of the pressure profile, the Galician player has a good repeatability of his control gesture between the four occurrences (Fig. 11). This observation is different from the previous ones made in the piece "Lolina" during which the control gesture was different for each repetition (Sec. V B).

This musical task necessitates an extreme variation of bag pressure. It imposes the control of the bag and forces the musician to synchronize his gesture with the music. To create a pause in the music, the player must lift his arm very quickly. The player needs to anticipate this specific movement, visible on the angular velocity of the arm. This imposes the arm movement for the entire phrase (Fig. 11). As mentioned previously, the bag must be empty enough in order to sufficiently decrease the bag pressure. This means that the player must fill the bag with air just after the pauses (Fig. 11). A suction effect appears when the bag pressure decreases abruptly, visible on the inflow measurement (Fig. 11). This effect might help to stop the reeds' oscillations.

In conclusion, it appears that performing pauses necessitates large and abrupt variations of the bag pressure. The bag pressure has to dip below the offset threshold of the entire set of pipes (drones and melodic pipe) during a short period of time. The achievement of this effect is facilitated by a small bag and a high offset threshold value, which are two conditions satisfied by the Galician bagpipe but not the Majorcan one (Table I). These considerations confirm once more that the organology of an instrument and its repertoire are closely linked.

\section{CONCLUSIONS AND PERSPECTIVES}

This paper presents an initial study of the bagpipe bag control through the comparison of control parameters (insufflation, bag pressure, and arm displacement) by pipers with different levels of expertise playing two different bagpipes. These two bagpipes, one Galician and one Majorcan, are similar in an organological point of view. They differ 

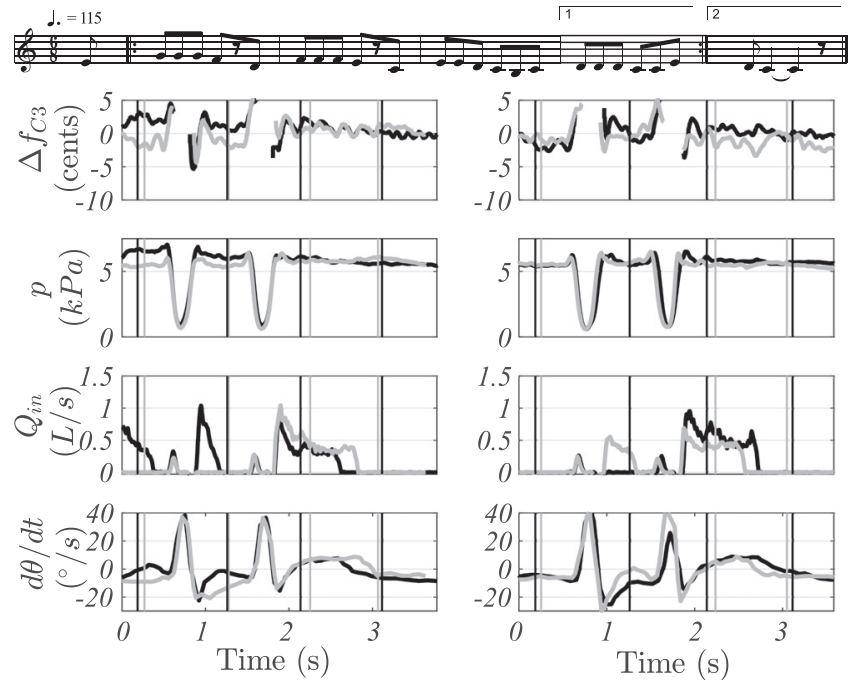

FIG. 11. Control parameters during a piece of music presenting pauses ("Roxelio," score on top) played by the Galician player B: the pitch variation of the drone around its mean frequency $\left(\Delta f_{C 3}\right)$, the bag pressure $(P)$, the inflow $\left(Q_{\text {in }}\right)$, and the angular velocity of the arm $(d \theta / d t)$. The repeated sentence appears twice in the musical piece: at the beginning (left) and at the end (right). Each time the first occurrence is in black and the second in gray. The vertical lines correspond to the bars.

essentially by their bag volume, much larger for the Majorcan bagpipe (Table I).

A basic mechanical model of the bag allowed the interpretation of the variation of the bag pressure as a consequence of the air supply of the bag and the arm displacement (Sec. II). Through this mechanical framework, the minimal control needed to produce the continuous sound characteristic for this type of instrument is easily interpreted. The bag pressure must be maintained within a small range $(5 \mathrm{kPa}<P$ $<6.5 \mathrm{kPa}$ ) through the precise coordination of the insufflations and the arm displacement (Sec. II), more or less accurately following the level of the player (Sec. V A).

The observation of the bag pressure in musical context shows that the players vary this pressure within the accepted range of ( $5 \mathrm{kPa}<P<6.5 \mathrm{kPa})$, varying according to the musical task with a good rate of repeatability (Sec. VB). Different control strategies are used for different bagpipes. Galician players maintain a regular insufflation/arm displacement rhythm all along the musical excerpt. They do not seem to synchronize their control gesture to the musical task and the evolution of the associated parameters is not necessarily the same for two iterations of the same phrase (Sec. V B).

On the other hand, the Majorcan players modify the rhythm and the coordination of their insufflations and arm displacements in relation to the musical tasks. In this case, a good repeatability of the control gesture is observed between two iterations of the same phrase by a given piper (Sec. V B). A similar kind of synchronization between the gesture and the musical task happens with Galician players when a very large bag pressure variation is needed, such as for creating pauses within the music (Sec. V C).

These two different strategies could be influenced by the different bag sizes of the instrument. The Majorcan bagpipe having a larger bag, the players must effectuate wider control variations to induce similar bag pressure variations.
From a broader point of view, the results of this study allow a better understanding of the supply system control of wind instruments during musical performances. The conclusions of this paper could lead to the same type of study on other musical instruments with an external supply system, such as the accordion, but also other wind instruments and the singing voice.

Further studies within a more ethnomusicological framework would put in perspective the perceived control strategies of the player with acoustic measurements. This would take into account varying levels of cultural specificities and the identification of control strategies that may be entirely musical, relating both to the instrument and to the musical culture in which the musician is embedded. Alongside this, further developments would include a more in-depth study of the behavior of the instrument, taking into account the wide variety of organological differences.

\section{ACKNOWLEDGMENTS}

The authors would like to thank the musicians who took part in the project: Álvaro Estévez Castro, Anxo Lorenzo, Gabriel Moya, Cristobal Prieto, Josep Rotger, Cándid Trujillo, and Miquel Tugores. This research was supported by Sorbone Universités program chaire GeAcMus and CONICYT through FONDECYT Project No.1161464.

${ }^{1}$ J.-P. Van Hees, Cornemuses: Un Infini Sonore (Coop Breizh, Spezet, France, 2014).

${ }^{2}$ W. A. Cocks, A. C. Baines, and R. D. Cannon, "Bagpipe," Grove Music Online, available at http://oxfordindex.oup.com/view/10.1093/gmo/ 9781561592630.article.01773 (Last viewed September 8, 2017).

${ }^{3}$ I. Cossette, B. Fabre, V. Fréour, N. Montgermont, and P. Monaco, "From breath to sound: Linking respiratory mechanics to aeroacoustic sound production in flutes," Acta Acust. Acust. 96, 654-667 (2010).

${ }^{4}$ C. Vauthrin, B. Fabre, and I. Cossette, "How does a flute player adapt his breathing and playing to musical tasks?," Acta Acust. Acust. 101, 224-237 (2015).

${ }^{5} \mathrm{~V}$. Fréour, "Acoustic and respiratory pressure control in brass instrument performance," Ph.D. thesis, McGill University, Montreal, Quebec, 2013.

${ }^{6}$ S. Carral, D. M. Campbell, and T. D. Rossing, "Relationship between blowing pressure, pitch, and timbre of a Scottish bellows blown border bagpipe," in Proceedings of the Stockholm Musical Acoustics Conference (2003), Vol. 1, pp. 251-254.

${ }^{7}$ R. B. Dannenberg, B. Brown, G. Zeglin, and R. Lupish, "McBlare: A robotic bagpipe player," in Proceedings of the 2005 Conference on New Interfaces for Musical Expression, National University of Singapore (2005), pp. 80-84.

${ }^{8}$ A. Almeida, C. Vergez, R. Caussé, and X. Rodet, "Physical study of doublereed instruments for application to sound-synthesis," in International Symposium in Musical Acoustics, (December 2002), Mexico, Mexico.

${ }^{9}$ J.-P. Dalmont, J. Gilbert, and S. Ollivier, "Nonlinear characteristics of single-reed instruments: Quasistatic volume flow and reed opening measurements," J. Acoust. Soc. Am. 114, 2253-2262 (2003).

${ }^{10}$ A. Almeida, C. Vergez, and R. Caussé, "Quasistatic nonlinear characteristics of double-reed instruments," J. Acoust. Soc. Am. 121(1), 536-546 (2007).

${ }^{11}$ A. de Cheveigné and H. Kawahara, "YIN, a fundamental frequency estimator for speech and music," J. Acoust. Soc. Am. 111(4), 1917-1930 (2002).

${ }^{12}$ S. McKerrell, "Sound Performing: Sound Aesthetics among Competitive Pipers," Int. Rev. Aesth. Sociol. Music 42, 165-187 (2011), available at http://www.jstor.org/stable/41228647?seq=1\#page_scan_tab_contents.

${ }^{13}$ B. Bergeot, A. Almeida, C. Vergez, and B. Gazengel, "Prediction of the dynamic oscillation threshold in a clarinet model with a linearly increasing blowing pressure,” Nonlin. Dyn. 73(1-2), 521-534 (2013).

${ }^{14}$ C. Balosso-Bardin, "The Mallorcan bagpipes (xeremies): An anthropological portrait of an instrument," Ph.D., SOAS University of London, London, 2016. 